\title{
Editorial
}

\section{Introduction to the Special Issue on Structured Light Coherence}

\author{
Olga Korotkova ${ }^{1, *}$ and Franco Gori ${ }^{2}$ \\ 1 Department of Physics, University of Miami, Coral Gables, FL 33146, USA \\ 2 Dipartimento di Ingegneria Industriale, Elettronica e Meccanica, Universita Roma Tre, 00146 Rome, Italy; \\ franco.gori@uniroma3.it \\ * Correspondence: o.korotkova@miami.edu
}

Citation: Korotkova, O.; Gori, F. Introduction to the Special Issue on Structured Light Coherence. Photonics 2021, 8, 457. https://doi.org/ $10.3390 /$ photonics 8100457

Received: 19 August 2021

Accepted: 8 October 2021

Published: 19 October 2021

Publisher's Note: MDPI stays neutral with regard to jurisdictional claims in published maps and institutional affiliations.

Copyright: (c) 2021 by the authors. Licensee MDPI, Basel, Switzerland. This article is an open access article distributed under the terms and conditions of the Creative Commons Attribution (CC BY) license (https:/ / creativecommons.org/licenses/by/ $4.0 /)$.
Statistical optics, and optical coherence in particular, developed into a stand-alone branch of physical optics in the second half of the 20th century and has found a number of ground-breaking applications in astronomical measurements, medical diagnostics, environmental remote sensing, and wireless communications [1]. The coherence states of optical sources were shown to play a fundamental role in the evolution of various measurable properties of light fields radiated by them, such as the diffraction rate, the spectral composition, the polarization properties, and the orbital angular momentum structure.

The design of optical radiation with a prescribed combination of its fundamental properties at a specified range from the source has become a feasible and affordable possibility for a modern optical laboratory. Thus, coherence theory has been added to the disciplines (essentially of coherent optics) contributing to the development of structured light [2]. This became possible largely because of the recent increase in the use of electrically controllable, spatially/temporally resolved, amplitude/phase spatial light modulators (or digital micromirror devices).

In 2007-2009, necessary and sufficient conditions for devising correct cross-spectral densities were found [3,4]. In particular, this was exploited for designing sources giving legitimate average intensity distributions in the far field. It was then realized that spatial structuring of the source coherence provides the direct tool for fine control of the far-field average intensity profiles. Using this approach, it was illustrated that stationary light sources with specially designed coherence states can radiate beams exhibiting various exotic types of behavior on propagation: they can self-focus, form rings, flat plateaus, split into multiple replicas, etc.

Light carrying structured coherence states were also applied to various imaging, communication, and remote sensing optical systems operating in vacuum, gradient-index fibers, atmospheric and oceanic turbulence, and biological tissues. In particular, tailoring the illumination's coherence state to match the object features was recently demonstrated as a power tool for improvement of the resolution in classic imaging systems. The coherence state structuring was also applied to the cyclo-stationary fields (random pulses) for controlling their average arrival distributions. The design of scattering media with structured correlation functions in the refractive index was also attempted. A plane wave was shown to scatter from such media to a far-field with a desired average intensity.

The special issue contains several papers in which the aforementioned phenomena are summarized, illustrated, and extended. The authors of [5] provide a mini-review of a unique class of stationary sources possessing a "twist phase". Such sources carry the orbital angular momentum and, in the case of axial asymmetry, they radiate beams rotating on propagation (even in free space). As applied to twisted sources, Bochner's theorem has provided remarkable new opportunities in twist strength and range control.

Of particular interest in statistical optics are sources radiating shape-invariant beams. In the class of stationary sources, the invariance must be met by both the average intensity and the degree of coherence of the source. The authors of [6] introduced a novel class of stationary beams, termed Laguerre-Christoffel-Darboux sources, that obey such characteristics. 
A source coherence state is generally a complex-valued function. While, in the absence of the phase, the radiated far field remains symmetric with respect to the optical axis, the non-trivial phase distribution will generally break this symmetry. For example, a remarkable effect of a radial acceleration (an airy beam-like effect) can be observed in stationary beams if the phase of the source coherence state is a nonlinear (cubic monomial related) function of point separation. The phase non-linearities substantially complicate the theoretical predictions.

In [7], the possibility of substituting a coherence state with a nonlinear phase by a linear combination of those with linear phases is suggested, a method that could be used for theoretical work and the design of elementary (linear) phase devices of correlation optics. Due to the recent wide-spread use of manipulation of optical fields with the high spatio-temporal resolution devices (e.g., spatial light modulators), it became possible not only to computer-simulate light fields with arbitrary statistics but also to implement them in real-light systems.

In [8], the possibility of fine control of the magnitude and the phase of a speckle-field statistics is achieved. Such manipulation is shown to produce entirely new classes or stationary fields with controllable statistical properties of different orders.

The extension of the structured coherence from scalar to vectorial domain can be formulated with the help of the generalized (two-point) Stokes parameters. The authors of [9] suggest a brief overview of this extension and discuss its practical implications.

The development of partially coherent sources with structured correlations is particularly useful for optical systems operating in random environments, such as atmospheric and oceanic turbulence. Due to their inherent multi-mode structure, they perform in a more robust manner as compared with their coherent analogs. In [10], a novel class of partially coherent beams is analyzed regarding its interaction with the maritime atmosphere as a particularly strong beam phase modifier at small spatial scales.

Cyclo-stationary pulses with separable, structured temporal and spatial coherence states are introduced in [11]. Such pulses can form the required two-dimensional spatial average intensity distributions with the finely adjusted average arrival time distribution. It is illustrated, in particular, that spatial and temporal counterparts of the source coherence function may come from different families and be adjusted independently.

The control of optical coherence states can also be achieved via the interference of plasmon waves. A numerical analysis is performed in [12] to illustrate the manipulation of light coherence on a metal plate with a suitably chosen array of holes. The associated phenomena of coherence bandgaps and resonances are also highlighted.

A wide class of structured-coherence sources has been introduced by the authors of [13] as continuous superpositions of standard Gaussian Schell-model sources with different parameters and suitable weights. It is shown that a particular type of superposition gives rise to cross-spectral densities that are expressible in terms of a Laplace transform, whose use is still rather uncommon in optical propagation problems. Furthermore, conditions are studied in order for the fields radiated from sources of this class to maintain their transverse shape upon paraxial propagation.

Funding: This research received no external funding.

Institutional Review Board Statement: Not applicable.

Informed Consent Statement: Not applicable.

Conflicts of Interest: The authors declare no conflict of interest.

\section{References}

1. Korotkova, O.; Gbur, G. Applications of Optical Coherence Theory. Prog. Opt. 2020, 65, 43-104.

2. Forbes, A. Structured Light: Tailored for Purpose. Opt. Photon. News 2020, 31, 24-31. [CrossRef]

3. Gori, F.; Santarsiero, M. Devising genuine spatial correlation functions. Opt. Lett. 2007, 32, 3531-3533. [CrossRef] [PubMed]

4. Martinez-Herrero, R.; Mejìas, P.M.; Gori, F., Genuine cross-spectral densities and pseudo-modal expansions. Opt. Lett. 2009, 34, 1399-1401. [CrossRef] [PubMed] 
5. Liu, Z.; Wan, L.; Zhou, Y.; Zhang, Y.; Zhao, D. Progress on Studies of Beams Carrying Twist. Photonics 2021, 8, 92. [CrossRef]

6. Martínez-Herrero, R.; Santarsiero, M.; Piquero, G.; González de Sande, J.C. A New Type of Shape-Invariant Beams with Structured Coherence: Laguerre-Christoffel-Darboux Beams. Photonics. Photonics 2021, 8, 134. [CrossRef]

7. Mei, Z.; Korotkova, O. Linear Combinations of the Complex Degrees of Coherence. Photonics 2021, 8, 146. [CrossRef]

8. Hyde, M.W. Independently Controlling Stochastic Field Realization Magnitude and Phase Statistics for the Construction of Novel Partially Coherent Sources. Photonics 2021, 8, 60. [CrossRef]

9. Setälä, T.; Saastamoinen, K.; Friberg, A.T. Coherence Stokes Parameters in the Description of Electromagnetic Coherence. Photonics 2021, 8, 85. [CrossRef]

10. Sun, B.; Lü, H.; Wu, D.; Wang, F.; Cai, Y. Propagation of a Modified Complex Lorentz-Gaussian-Correlated Beam in a Marine Atmosphere. Photonics 2021, 8, 82. [CrossRef]

11. Ding, C.; Korotkova, O.; Horoshko, D.; Zhao, Z.; Pan, L. Evolution of Spatiotemporal Intensity of Partially Coherent Pulsed Beams with Spatial Cosine-Gaussian and Temporal Laguerre-Gaussian Correlations in Still Water. Photonics 2021, 8, 102. [CrossRef]

12. Gbur, G.; Smith, M. Controlled Coherence Plasmonic Light Sources. Photonics 2021, 8, 268. [CrossRef]

13. Gori, F.; Santarsiero, M. Variant-coherence Gaussian Sources. Photonics 2021, 8, 403. [CrossRef] 